\title{
Quorum sensing of Streptococcus mutans is activated by Aggregatibacter actinomycetemcomitans and by the periodontal microbiome
}

Szymon P. Szafrański ${ }^{1,4}$, Zhi-Luo Deng ${ }^{1}$, Jürgen Tomasch', Michael Jarek², Sabin Bhuju², Manfred Rohde 3 , Helena Sztajer ${ }^{1 \dagger}$ and Irene Wagner-Döbler ${ }^{1 *+}$

\begin{abstract}
Background: The oral cavity is inhabited by complex microbial communities forming biofilms that can cause caries and periodontitis. Cell-cell communication might play an important role in modulating the physiologies of individual species, but evidence so far is limited.

Results: Here we demonstrate that a pathogen of the oral cavity, Aggregatibacter actinomycetemcomitans (A. act.), triggers expression of the quorum sensing (QS) regulon of Streptococcus mutans, a well-studied model organism for cariogenic streptococci, in dual-species biofilms grown on artificial saliva. The gene for the synthesis of the QS signal XIP is essential for this interaction. Transcriptome sequencing of biofilms revealed that $S$. mutans up-regulated the complete QS regulon (transformasome and mutacins) in the presence of $A$. act. and down-regulated oxidative stress related genes. A.act. required the presence of $S$. mutans for growth. Fimbriae and toxins were its most highly expressed genes and up-regulation of anaerobic metabolism, chaperones and iron acquisition genes was observed in co-culture. Metatranscriptomes from periodontal pockets showed highly variable levels of S. mutans and low levels of A. act.. Transcripts of the alternative sigma-factor SigX, the key regulator of QS in S. mutans, were significantly enriched in periodontal pockets compared to single cultures $\left(\log _{2} 4.159, F D R \leq 0.001\right.$, and expression of mutacin related genes and transformasome components could be detected.
\end{abstract}

Conclusion: The data show that the complete QS regulon of S. mutans can be induced by an unrelated oral pathogen and S. mutans may be competent in oral biofilms in vivo.

\section{Background}

Streptococcus mutans (S. mutans) has been isolated from caries lesions in 1924 and was consequently considered the etiological agent of caries, consistent with Koch`s postulates [1]. Its physiological properties were since then analysed in great detail and were in accordance with a role as primary causative agent of cavities [2]. Cultivation independent studies, particularly metagenome and metatranscriptome analyses, have completely revolutionized our understanding of oral diseases like caries and periodontitis.

\footnotetext{
* Correspondence: Irene.Wagner-Doebler@helmholtz-hzi.de

${ }^{\dagger}$ Equal contributors

'Microbial Communication, Helmholtz-Center for Infection Research,

Braunschweig, Germany

Full list of author information is available at the end of the article
}

They clearly show that $S$. mutans is only a tiny component of the active oral microbiome. Even in caries lesions, it accounts for less than $1 \%$ of the active microorganisms $[3,4]$ and the onset of early childhood caries can be predicted by the presence of Prevotella spp. but not S. mutans [5]. The etiology of caries is therefore one of the prime examples for the paradigm shift from Koch's postulates towards the concept of a polymicrobial origin of disease caused by a shift in the composition and metabolic activity of a complex community towards dysbiosis [6]. It is thus important to understand the mechanisms that contribute to dysbiosis development and this will provide novel concepts for treatment [6]. For example, modifying the interaction pattern could potentially have complex downstream effects on the community [7]. 
Cell-cell communication might be one of the mechanisms that can tip the balance between health and dysbiosis [8]. Oral biofilms are hot spots of cell-cell communication due to the high species diversity, the physical contact between adjacent cells, and large metabolic activity of the community [9]. S. mutans is a good model organism for studying the role of QS in oral biofilms because the functioning of its QS system is relatively deeply understood and some of its characteristics are conserved. In streptococci, short hydrophobic peptides induce competence via a RGG type transriptional regulator [10]. These peptides have been shown to mediate interspecies communication [11], and have been exploited for antibacterial strategies $[7,12,13]$. Communication can be induced by environmental signals and change the resistance of the bacteria towards cell wall degrading lysozyme [14]. However, it is currently not known if streptococci, or S. mutans in particular, are competent in their natural habitats in vivo, i.e. in the human or animal host. Metagenomics is not informative in this respect, since it only shows the presence of the DNA, but competence is a strongly regulated phenotype. Sequencing of the total mRNA of the microbial community provides information which genes $S$. mutans is actively transcribing in vivo. Therefore we searched the published metatranscriptomes of periodontal pockets [15-18] for transcripts from S. mutans and A. act. and investigated if the competence regulator SigX and downstream genes are expressed in the natural biofilm under the prevailing conditions and in the presence of the complex oral microbial community.

S. mutans synthesizes two quorum sensing (QS) signals, competence inducing peptide (CSP) and sigX inducing peptide (XIP) that are sensed through a two-component signal system (ComDE) and a Rgg-type intracellular transcriptional regulator (ComR), respectively [19]. Upon activation of ComR through binding of XIP, transcription of the alternative sigma-factor SigX is induced [19]. We recently showed that CSP controls transcription of mutacins only, thus we suggest to rename it mutacin inducing peptide (MIP) [20]. The regulon of SigX in S. mutans includes not only the transformasome machinery mediating genetic competence, but also mutacins whose transcription is controlled by the ComE response regulator [20]. Thus SigX is the central regulator of QS in $S$. mutans. It is not known if $\operatorname{sig} X$ is expressed in vivo. Moreover, natural conditions under which MIP is synthesized are unknown. We previously demonstrated induction of $\operatorname{sig} X$ by the human pathogenic fungus Candida albicans; both transformasome and mutacins were upregulated in co-culture, in accordance with the central role of SigX in S. mutans [20,21].

Here we now ask if inter-kingdom communication between $S$. mutans and C. albicans is an exception, or if $S$. mutans might be able to communicate with other members of the oral microbiome. We studied its interaction with Aggregatibacter actinomycetemcomitans (A. act). This pathogen has been regarded as etiological agent of aggressive periodontitis for decades, but today is found to be a minor component of the oral cavity [22] while periodontitis is regarded as a polymicrobial infection [23], similar to the situation with S. mutans and dental caries. For species to interact in the oral cavity, their co-localization in vivo is essential. Only recently has it been possible to observe such co-localization with genus-level taxonomic resolution. In dental plaque samples, interestingly, Aggregatibacter spp. and Streptococcus spp. were found as dense aggregates at the tips of abundant hedgehog structures [24]. Thus studying interactions between S. mutans and $A$. act. might reveal mechanisms contributing to biofilm development in the oral cavity.

A. act. is doomed to co-operate. It entirely depends on other oral microorganisms to grow in saliva [25]. A "food for detoxification" interaction has been unraveled between A. act. and the commensal S. gordonii [26]. S. gordonii excretes lactic acid, which $A$. act. uses as a carbon source, and hydrogen peroxide, which $A$. act. detoxifies, and in a murine abcess model both species are more virulent together than alone [27]. Cocultivation with S. gordonii triggers expression of the complement resistance protein ApiA in A. act. and in such a way increases its resistance to host innate immunity [28].

Here we analysed the interaction between $S$. mutans and $A$. act. in dual-species biofilms. We monitored induction of SigX of $S$. mutans using a $P_{\text {sigX }}$-GFP reporter strain [29]. To determine through which route $A$. act. might communicate with $S$. mutans we deleted key genes of $S$. mutans. We analysed the transcriptomes of both species using RNA sequencing. Finally we asked if competence and mutacin synthesis might be expressed by $S$. mutans also in oral biofilms in vivo. To this end we extracted its transcripts from metatranscriptomes of periodontal pockets and analysed the expression of the SigX regulon.

\section{Methods}

Strains, culture conditions and media

Microorganisms used in this study are listed in Additional file 1. A. act. ATCC 33384 was used in all experiments except for RNA sequencing where the "rough" strain HK1651 (CCUG 56173, Göteborg, Sweden) was used because it has a fully assembled and annotated genome. All cultures were incubated aerobically at $37{ }^{\circ} \mathrm{C}$ with $5 \% \mathrm{CO}_{2}$ without shaking. Pre-cultures of $S$. mutans and A. act. strains were grown in Todd-Hewitt broth supplemented with yeast extract (THBY) (Becton, Dickinson and Company, Sparks, MD, USA). For the sigX reporter strain and deletion mutants, erythromycin $\left(3 \mu \mathrm{g} \mathrm{ml}{ }^{-1}\right.$ and $10 \mu \mathrm{g} \mathrm{ml}^{-1}$, respectively) was added. Biofilms were cultivated in YNBB2 medium which contains $6.7 \mathrm{~g} \mathrm{l}^{-1}$ yeast nitrogen base (YNB) synthetic medium (Difco Laboratories, Detroit, Mi, USA), $75 \mathrm{mM} \mathrm{Na} \mathrm{HPO}_{4}-\mathrm{NaH}_{2} \mathrm{PO}_{4}$ 
(pH 7.3), casamino acids ( $2 \mathrm{~g} \mathrm{l}^{-1}$, Becton, Dickinson and Company) and sucrose $\left(5 \mathrm{~g} \mathrm{l}^{-1}\right)$. The $\mathrm{pH}$ during biofilm growth was in the range 7.0-6.4. Biofilms grown on modified chemically defined artificial saliva medium (artificial saliva) [30] were used for RNA sequencing. Mucin was omitted from the medium, haemin was replaced with $1.2 \mu \mathrm{M} \mathrm{FeCl}_{3}, 14.6 \mathrm{mM}$ sucrose was added and the medium was buffered with $75 \mathrm{mM} \mathrm{Na}_{2} \mathrm{HPO} 4-$ $\mathrm{NaH}_{2} \mathrm{PO}_{4}$ ( $\mathrm{pH}$ 7.3). In some experiments, chemically defined mineral medium CDM [31] was used. For the construction of the hdrRM, brsRM and lias deficient strains, the PCR ligation mutagenesis approach [32] was used. Primers are listed in Additional file 1: Table S1. Mutants were verified by PCR and sequencing.

\section{Biofilm analysis}

Crystal violet staining of biofilms, DAPI staining, fluorescence microscopic analysis and field emission scanning electron microscopy were performed as described [21]. For quantitative PCR, extracellular DNA was removed from the biofilms, genomic DNA was extracted and $S$. mutans genomes were quantified as described [21]. Quantification of $A$. act. genomes was performed using forward primer GGACGGGTGAGTAATGCTTG and reverse primer CCTTTACCCCACCAACTACC and the annealing temperature was $58{ }^{\circ} \mathrm{C}$ (modified from [33]). To convert nanograms of DNA to numbers of cells, $2.222 \mathrm{fg}$ was used as the weight of the genome of A. act.

\section{Preparation of conditioned media and reporter strain biofilm assay}

Culture supernatants were withdrawn, centrifuged (5000 rpm, $\left.20 \mathrm{~min}, 4{ }^{\circ} \mathrm{C}\right)$, sterile filtered $(0.22 \mu \mathrm{m}$, Roth, Karlsruhe, Germany) and frozen at $-20{ }^{\circ} \mathrm{C}$. S. mutans reporter strain $\mathrm{SMP}_{\text {sigX }} \mathrm{GFP}$ was grown in black Nunc 96 Well Optical Bottom Plates (Nunc, Langenselbold, Germany) in YNBB2 medium for $8 \mathrm{~h}$. Cultivation media were removed and conditioned media (warmed to $37{ }^{\circ} \mathrm{C}$ ) were loaded onto the reporter strain biofilms. Biofilms were further incubated for $3 \mathrm{~h}$, supernatants were removed and the induction of $\operatorname{sig} X$ was measured as described [21].

\section{RNA isolation and sequencing}

Biofilms of S. mutans, A. act. or both were cultivated in 24 well cell culture plates (Greiner Bio-One, Frickenhausen, Germany) for 4 to $24 \mathrm{~h}$. After removal of the supernatants, biofilms were covered with $300 \mu \mathrm{l}$ RNA Protect (Qiagen, Hilden, Germany). The biofilms were scraped off with sterile one-way cell scrapers, transferred to sterile Eppendorf tubes, centrifuged $\left(4{ }^{\circ} \mathrm{C}, 5 \mathrm{~min}, 13,000 \mathrm{rpm}\right)$, the supernatant was removed and the pellets were stored at $-70{ }^{\circ} \mathrm{C}$ until RNA extraction. For RNA extraction the pellets were suspended in $0.2 \mathrm{ml}$ lysis buffer containing $10 \mathrm{mM}$ Tris, $1 \mathrm{mM}$ EDTA (pH 8.0), $2.5 \mathrm{mg} / \mathrm{ml}$ lysozyme (Sigma-Aldrich, Taufkirchen, Germany) and 50 $\mathrm{U} / \mathrm{ml}$ mutanolysin (Sigma-Aldrich, Taufkirchen, Germany) and incubated at $25^{\circ} \mathrm{C}$ for $1.5 \mathrm{~h}$ at $350 \mathrm{rpm}$. Subsequently $1 \mathrm{ml}$ TRIzol reagent (Invitrogen, Carlsbad, CA, USA) was added, samples were mixed and incubated at room temperature (RT) for $5 \mathrm{~min}$. Cells were then vortexed for $30 \mathrm{~s}$ in the presence of $50 \mu \mathrm{g}$ sterile, acid-washed glass beads (diameter $106 \mu \mathrm{m}$; Sigma-Aldrich, Taufkirchen, Germany). This was repeated 10 times with 1 min intervals on ice. Samples were briefly centrifuged, supernatants were transferred to new tubes and vortexed with ice cold 1-bromo-3-chloropropane (Sigma-Aldrich, Taufkirchen, Germany) for $15 \mathrm{~s}$ followed by $5 \mathrm{~min}$ incubation at RT. Samples were centrifuged $\left(4{ }^{\circ} \mathrm{C}, 15 \mathrm{~min}, 13,000 \mathrm{rpm}\right)$, the aqueous phase was collected, nucleic acids were precipitated with 1 volume of ice cold isopropanol, the solvent was removed and the pellet was suspended in double distilled autoclaved water. After removal of DNA with RNase free DNaseI (Qiagen, Hilden, Germany) using the in-solution digestion protocol, RNA was purified using the RNeasy mini kit (Qiagen, Hilden, Germany) according to the manufacturer's instructions with additional DNaseI on-column digestion. Bacterial rRNAs were depleted using MicrobExpress (Ambion, Austin, TX). mRNA was converted to cDNA using ScriptSeq v2 RNA-Seq (Epicentre Biotechnologies, Madison, Wisconsin, USA). For sequencing $12 \mathrm{pM}$ of each library was used and 8 samples were multiplexed on a single lane. Cluster generation was performed with cBot (Illumina) using a TruSeq SR Cluster Kit v3-cBot-HS (Illumina). Two biological replicas were sequenced for each treatment. In total two and half lanes (containing 20 libraries) were single-end sequenced for 50 cycles on an Illumina HiSeq 2500 sequencer using the TruSeq SBS Kit v3 - HS (Illumina). Image analysis and base calling were performed using the Illumina pipeline v 1.8. Sequencing data are available at Gene Expression Omnibus under accession number GSE75019.

\section{RNAseq data analysis}

The S. mutans UA159 and A. act. HK1651 reference genomes (GenBank accession numbers NC_004350.2 and CP007502.1, respectively) were used. After clipping adapters and barcodes, reads were aligned with the reference genome using Bowtie 2 [34]. HTSeq-count was used to count the mapped reads per gene (http://www-huber. embl.de/users/anders/HTSeq). Differential gene expression was calculated using the $\mathrm{R}$ package edgeR [35]. Genes showing differential expression in dual-species biofilms (all time points compared to the $4 \mathrm{~h}$ time point, $\log _{2}$ fold change $<-2$ and $>2 ; p<0.01$, were sorted into clusters using the c-means algorithm [36]. The Rockhopper software was used to identify novel transcripts and determine 
operons [37]. PRIMER \& PERMANOVA+, (PRIMER-E, Plymouth, UK) were used to perform PCA analysis based on standardized log-transformed sequence abundances grouped to genes. The $\mathrm{R}$ package pheatmap was used to create heatmaps [38].

\section{Results}

Induction of the alternative sigma-factor SigX of $S$. mutans by $A$. act

To determine if A.act. is able to induce QS in S. mutans we co-cultured it with a reporter strain. S. mutans $\mathrm{SMP}_{\text {sig }} \mathrm{GFP}$ carries a plasmid where the promoter of the alternative sigma-factor $\operatorname{sig} X$, the master regulator of both competence and mutacin synthesis, is fused to GFP [29]. Green fluorescence was measured during $24 \mathrm{~h}$ of biofilm growth. In the absence of a co-cultivation partner, no induction of fluorescence was observed in S. mutans (Fig. 1a). By adding the autoinducer XIP (sigX inducing peptide), strong fluorescence could be induced (Fig. 2a), indicating that extracellular XIP could be imported into the cells in this medium [39]. Live, but not heat inactivated A. act. strongly induced sigX expression in dual-species biofilms (Fig. 1a). Fluorescence showed a peak after $10 \mathrm{~h}$ of biofilm growth and declined subsequently. Quantification of $\operatorname{sig} X$ expression by q-RT-PCR (Fig. 1b) similarly showed a peak at $10 \mathrm{~h}$ in co-culture.
Both species grew better together than alone. Biofilm biomass increased continuously for $S$. mutans biofilms, but much stronger in the dual-species biofilm of $S$. mutans with $A$. act (Fig. 1c). At every time point the biomass of dualspecies biofilms was at least twice that of single-species biofilms. A.act. did not form biofilms when grown alone, only small cell aggregates attached to the bottom of the wells were observed. q-PCR of the $16 \mathrm{~S}$ rRNA gene revealed that cell numbers of $S$. mutans increased throughout the experiment and reached $\sim 2 \times 10^{8}$ cells/well in mono-culture and more than twice that amount $\left(\sim 4.5 \times 10^{8}\right.$ cells/well $)$ in dual-species biofilms (Fig. 1d). A.act. reached $\sim 6-8 \times 10^{8}$ cells/well in dual-species biofilms, but did not grow in single culture (Fig. 1e). The numbers of A.act. did not change significantly in dual species biofilms during the 6-12 h period. This implies that the increase in biofilm biomass during this time frame (Fig. 1c) corresponded to the increase in numbers and extracellular polysaccharides (EPS) of S. mutans.

The alternative sigma-factor SigX is activated by an extracellular compound in dual-species biofilms

To test whether a compound excreted into the medium might be responsible for the induction of $\operatorname{sig} X$ we applied sterile-filtered supernatants from single- and dual-species biofilms (cultivated for 4 and $24 \mathrm{~h}$ ) to biofilms of the $S$.

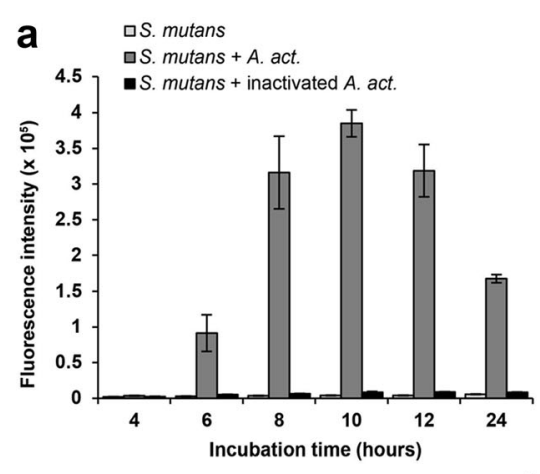

C

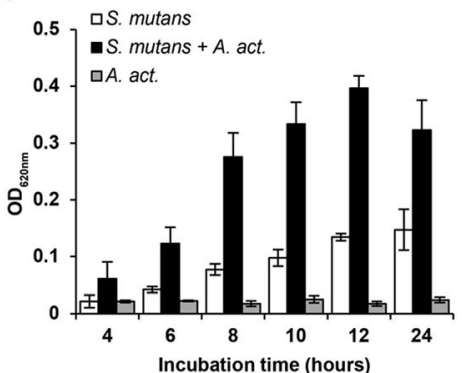

d
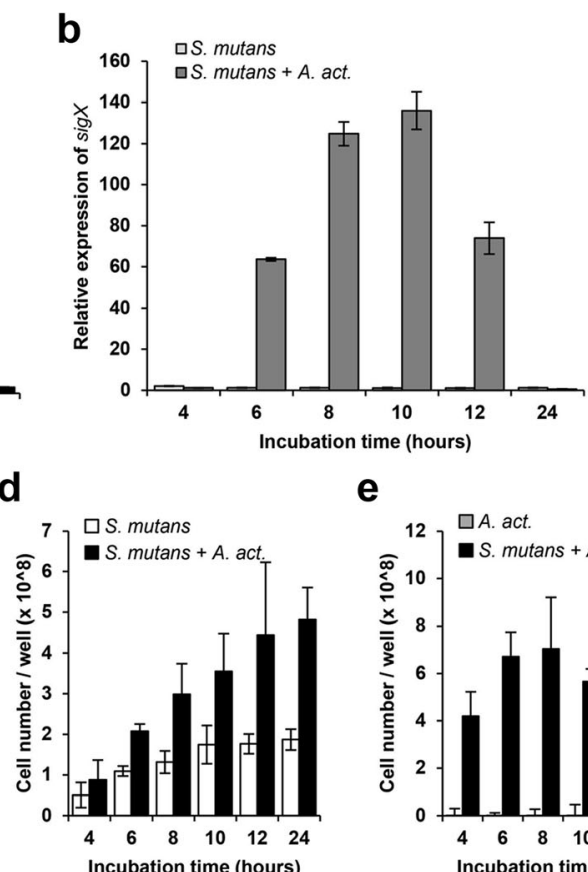

e

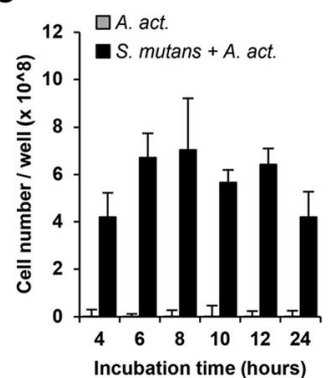

Fig. 1 The alternative sigma-factor sigX of S. mutans is induced by A. act. when both species are co-cultured in a biofilm. a sigX expression determined by fluorescence intensity of S. mutans SMP sigx GFP, a gfp-reporter for sigX expression. $\mathbf{b}$ sigX expression determined by q-RT-PCR. The data were normalized to sigX expression in a S. mutans biofilm after $4 \mathrm{~h}$. c Growth of S. mutans and A. act. in single- and dual-species biofilms determined by crystal violet staining. d Cell numbers of S. mutans and (e) A. act in single- and dual-species biofilms determined by q-PCR of the 165 rRNA gene. Mean and standard deviation of three independent experiments are shown for $\mathbf{a}-\mathbf{c}$, and of four independent experiments with two technical replicates for $\mathbf{d}-\mathbf{e}$ 

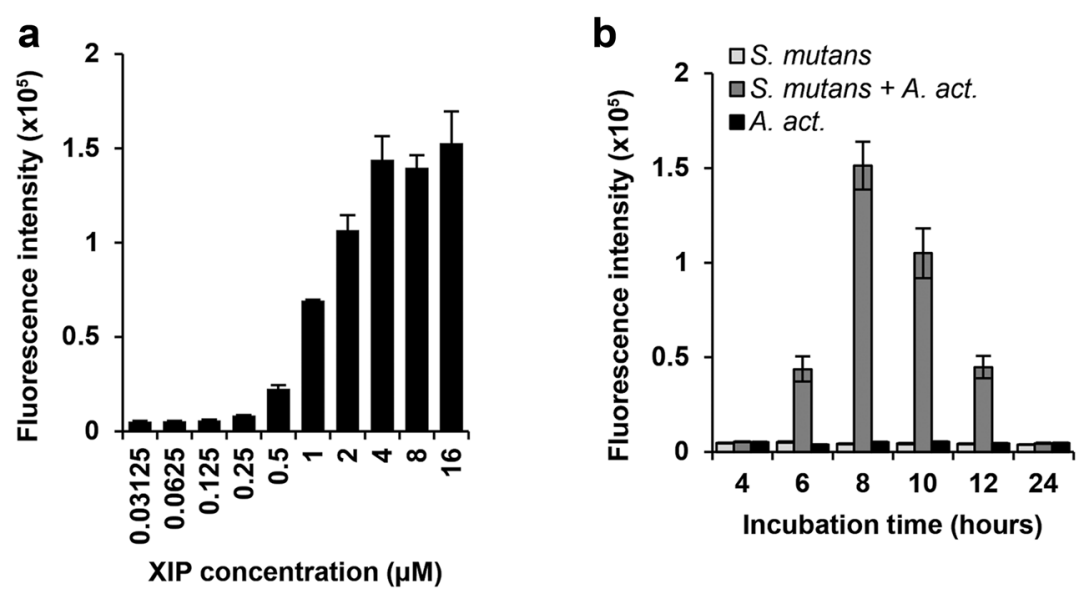

C

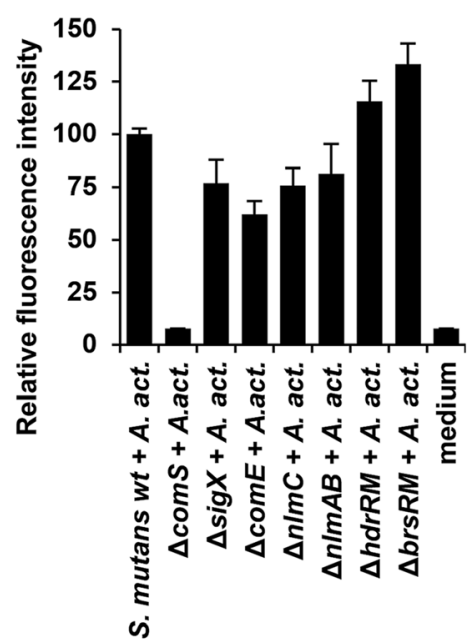

d
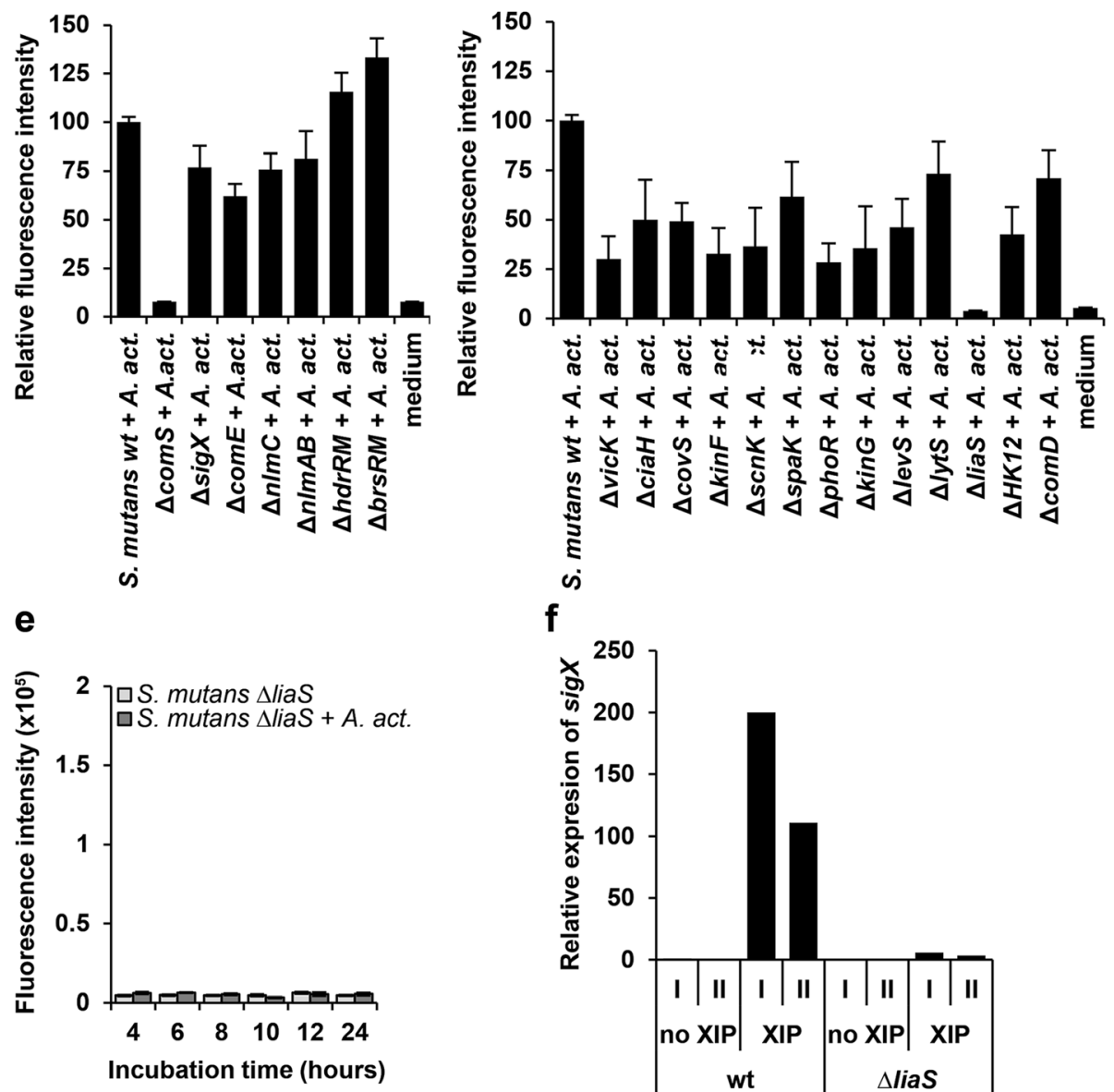

f

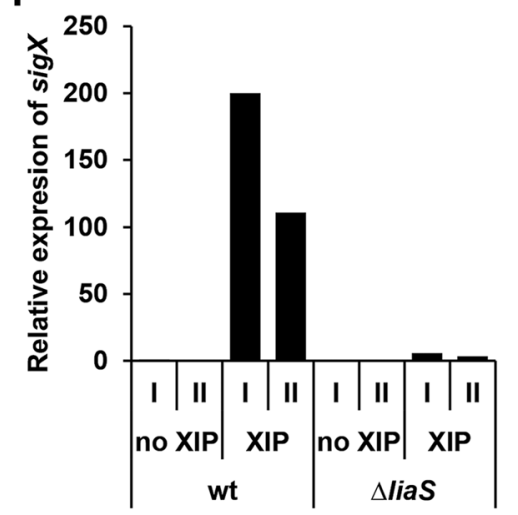

Fig. 2 (See legend on next page.) 


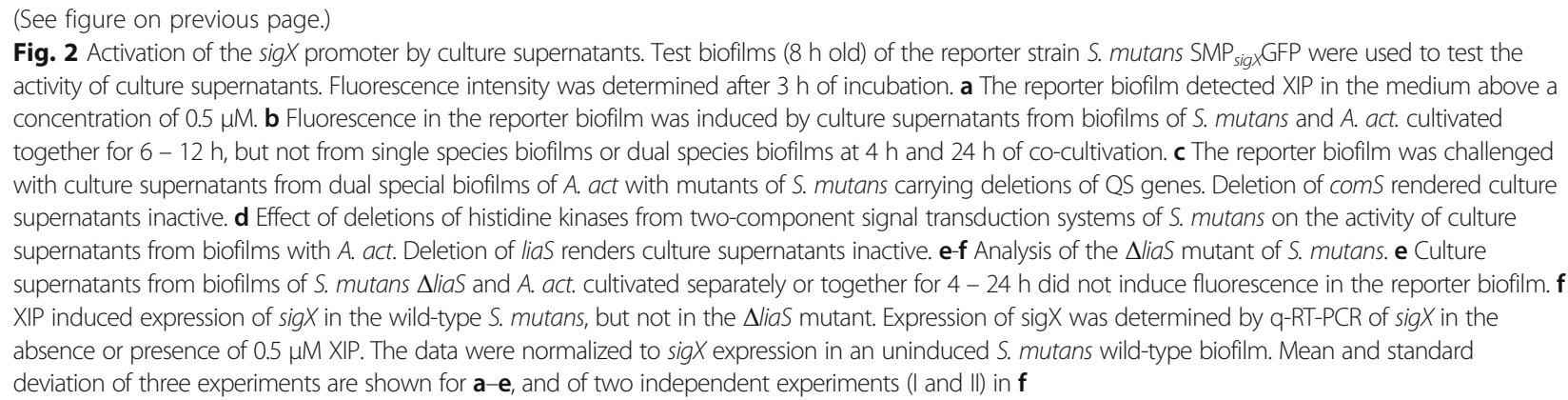

mutans $\mathrm{SMP}_{\text {sigX }} \mathrm{GFP}$ reporter strain grown for $8 \mathrm{~h}$; fluorescence intensity was determined after additional $3 \mathrm{~h}$ of incubation. Under these conditions, the reporter strain detected XIP concentrations above $0.25 \mu \mathrm{M}$ (Fig. 2a). Supernatants of dual-species biofilms that had been cultivated between 6 and $12 \mathrm{~h}$ activated $\mathrm{P}_{\text {sigX }}$, whereas mono-culture supernatants had no effect, irrespective of how long they had been cultivated (Fig. 2b). The data show that it is a component in the culture supernatant that activates the $\operatorname{sig} X$ promoter, and that this component is not present in single species biofilm supernatants of either $S$. mutans or A. act. Therefore, it must be the result of an interaction between those two organisms. The time-course of activation of the reporter strain by dual-species culture supernatants is in complete agreement with the time course of fluorescence and $\operatorname{sig} X$ expression observed in dual-species biofilms (Fig. 1a, b).

\section{Activation of SigX requires the autoinducer synthase Coms from S. mutans}

To investigate which regulatory systems of $S$. mutans might be involved in mediating the induction of $\operatorname{sig} X$, we tested culture supernatants from deletion mutants of $S$. mutans (Additional file 1) co-cultivated with A. act.. Fig. 2c shows that in the absence of the autoinducer synthase ComS, an active compound could not be produced in dual-species biofilms. ComS synthesizes a pre-peptide that is cleaved and exported to yield the mature XIP autoinducer. By contrast, neither SigX nor the response regulator ComE and the mutacins $\mathrm{NlmC}$ and $\mathrm{NlmAB}$ are required to produce an active supernatant in co-culture with $A$. act.. Interestingly, the activity of the supernatants was slightly higher in biofilms of $A$. act. with $S$. mutans lacking the two gene regulatory systems $H d r R M$ or BrsRM.

The data suggest that the autoinducer synthase ComS of $S$. mutans is stimulated by an extracellular component produced in co-culture with $A$. act. to synthesize the XIP pre-peptide, which after export and cleavage needs to be imported again and can then induce transcription of $\operatorname{sig} X$ and downstream genes. Since this re-import of XIP in S. mutans is abolished in rich medium [39], we tested the influence of different media on the induction of $\operatorname{sig} X$ in dual-species biofilms. SigX induction was only observed in peptide-free CDMS medium and in YNBB2, but not in THBYS or artificial saliva (AS) (Additional file 2), although the biofilms grew on all media (Additional file 3). Interestingly, mucin inhibited $\operatorname{sig} X$ activation in a concentration dependent manner (Additional file 4a). It was therefore omitted from the artificial saliva medium used for transcriptome analysis.

\section{Activation of SigX requires the histidine kinase liaS from S. mutans}

We then tested the influence of the histidine kinases (HKs) from the 13 two-component signal transduction systems of $S$. mutans on the activity of dual-species biofilm supernatants. Supernatants from $A$. act. grown in dual-species biofilms with deletion mutants of $S$. mutans lacking HKs were less active than those from $S$. mutans wild-type, most likely because the mutants have growth defects [40], but with one exception all of them were active (Fig. 2d). However, the supernatant from the $\Delta$ liaS mutant dual species biofilm was inactive over the full $24 \mathrm{~h}$ of growth with $A$. act. (Fig. 2e). This was not caused by impaired growth (data not shown). Subsequently, we tested the response of S. mutans $\Delta$ liaS to exogenous XIP. Mono-species biofilms of wild-type and S. mutans $\Delta$ liaS were cultivated with and without XIP for $8 \mathrm{~h}$ and the expression of the $\operatorname{sig} X$ gene was investigated using q-RT-PCR. In the wild-type, the expression of $\operatorname{sig} X$ was 200 fold or 100 fold induced by XIP, while it was only 2-5fold induced in the $\Delta$ liaS mutant (Fig. 2f), suggesting that this mutant is unable to import the XIP signal from the extracellular medium into the cell.

\section{Transcriptome analysis of S. mutans and A. act in dual- species biofilms}

We applied RNA sequencing to investigate the transcriptional activities of both $S$. mutans and $A$. act in dual species biofilms. Modified artificial saliva was used as cultivation medium to mimick the conditions in the oral cavity. We used the highly leukotoxic and adhesive "rough" strain A. act. HK1651 [41] because its fully annotated genome is available. It differs in terms of 
serotype, morphotype and amount of toxins produced from the type strain. HK1651 grew together with S. mutans in a similar way as the type strain of $A$. act. and it also activated $\operatorname{sig} X$, although with a slightly shorter duration in comparison to the type strain (Additional file 4b,c).

We analyzed the transcriptome of both species in dual-species biofilms during $(8 \mathrm{~h})$ and after $(24 \mathrm{~h})$ activation of $\operatorname{sig} X$ in comparison to gene expression in monospecies biofilms. In addition the transcriptomes of both species in dual-species biofilms were analyzed at $6,8,10$, 12 and $24 \mathrm{~h}$ of growth and compared to the expression at $4 \mathrm{~h}$. In total, 513.4 million reads were obtained, providing on average 11 and 1.1 million reads per sample. 93\% (S. mutans) and 84\% (A. act.) of the expressed genes had a mean count per gene per million reads (CPM) higher than 10 (Additional file 4d-f). Using Rockhopper we identified 434 operons and predicted 204 small RNAs (including 71 antisense RNAs) in the $S$. mutans genome (Additional file 5). Principal Component Analysis (PCA) (Fig. 3) showed a good correlation between replicates and clearly separated single- and dual-species biofilm transcriptomes. Moreover, gradual changes in co-culture transcriptomes of each species could be observed during the $24 \mathrm{~h}$ of growth and expression profiles could be separated into several clusters clusters and groups of clusters (Additional files 6 and 7).

\section{Comparison of transcriptomes of $S$. mutans grown alone and with $A$. act}

Comparative analysis of biofilm transcriptomes of $S$. mutans grown alone and with $A$. act. yielded 260 and
246 differentially expressed genes ( $\log _{2}$ fold change $\leq$ 2 and $\geq 2 ; p<0.01$ ) for the $8 \mathrm{~h}$ and $24 \mathrm{~h}$ time points, respectively. The complete SigX regulon was induced in dual-species biofilms, including the DNA uptake apparatus (Fig. 4a) and the recombination machinery (Fig. 4b) suggesting that in co-culture S. mutans became genetically competent. The alternative sigmafactor $\operatorname{sig} X$, the central regulator of QS, was among the most abundant transcripts and revealed a 72 fold change at $8 \mathrm{~h}$ (Fig. 4c). Accordingly, its primary target, the gene encoding the pre-peptide synthase ComS, was also highly upregulated. The ComDE twocomponent system that directly controls mutacin synthesis was slightly up-regulated, while the QS modulating $\mathrm{CiaRH}$ and the membrane bound protease HtrA were down-regulated (Fig. 4c). Mutacins also belong to the SigX regulon [20] and accordingly they were slightly up-regulated (Fig. 4d). Oxidative stress related genes, e.g. superoxide dismutase and chaperones, were repressed (Fig. 4e). Of the genes related to biofilm formation and adhesion, e.g. glucan binding proteins, some were up- and some were down-regulated (Fig. 4f). Carbohydrate metabolism of $S$. mutans most likely differed in the co-culture. Genes coding for exo-fructosidases, alpha-glucosidase and multi-sugar utilization enzymes (Fig. 4g) as well as sugar transport systems (Fig. 4h) were induced. Glycogen synthesis was induced especially at the later time-points (Fig. 4i). The expression of pyruvate dehydrogenase decreased in $8 \mathrm{~h}$ co-culture and was strongly induced at $24 \mathrm{~h}$ (Fig. $4 \mathrm{j})$.
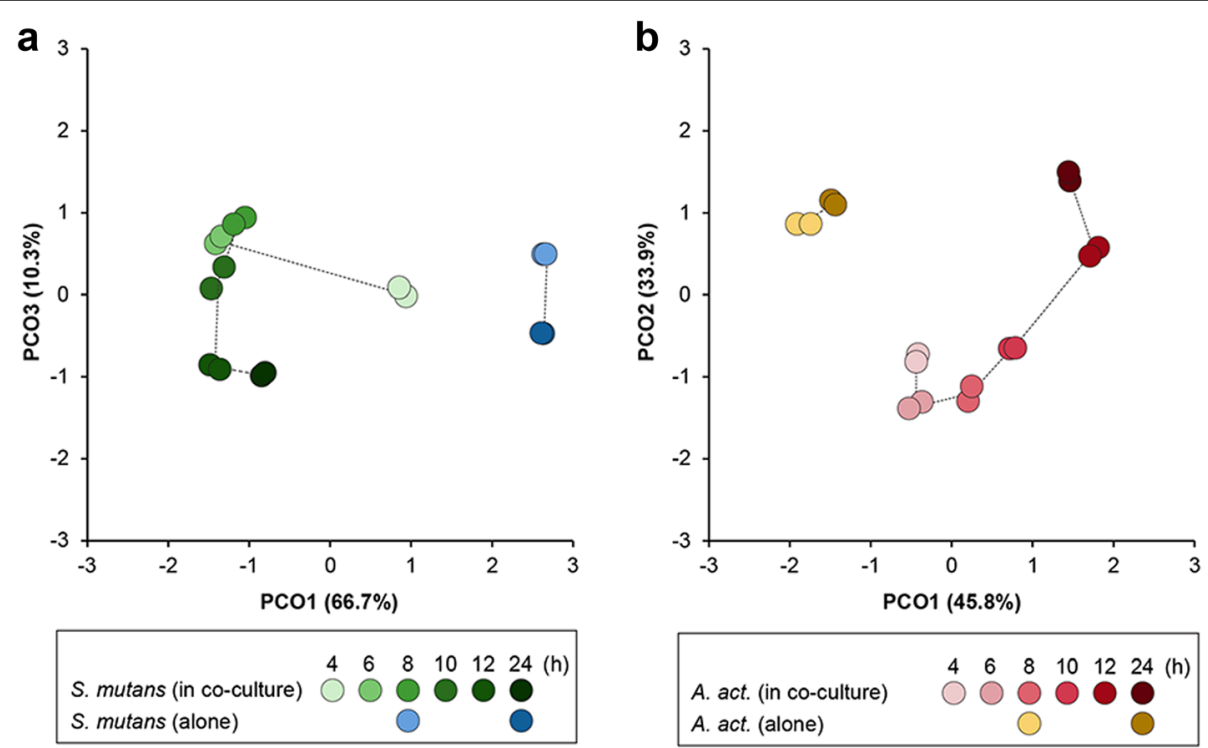

Fig. 3 Principal Component Analysis of single- and dual-species biofilm transcriptomes. a Transcriptomes of S. mutans grown in single-species biofilms (blue) and in dual-species biofilms with A. act. (green). b Transcriptomes of A. act. in single-species biofilms (red) and in dual-species biofilms with $S$. mutans (yellow). Standardized $\log _{2}$-transformed read counts were used in these analyses 


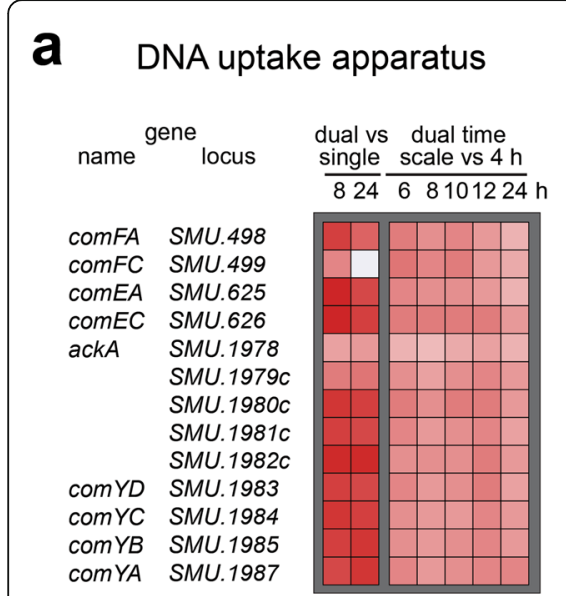

b DNA recombination and repair, other competence-related

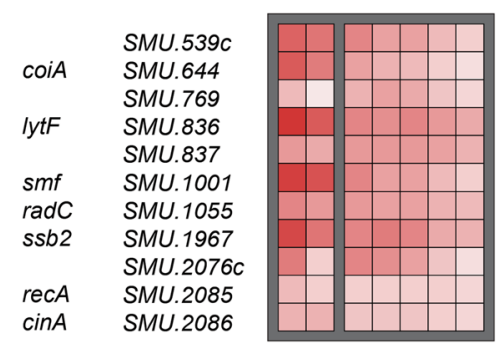

\section{Regulation of}

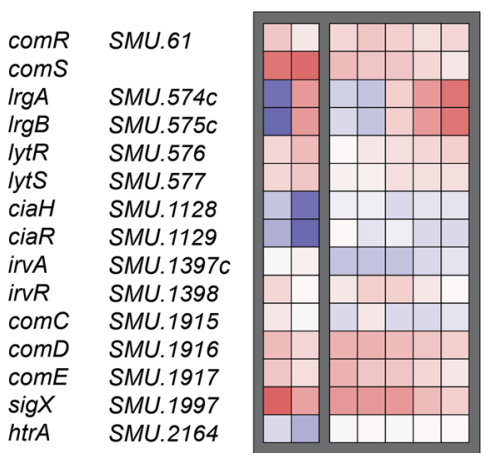

d

\section{Bacteriocins and related}

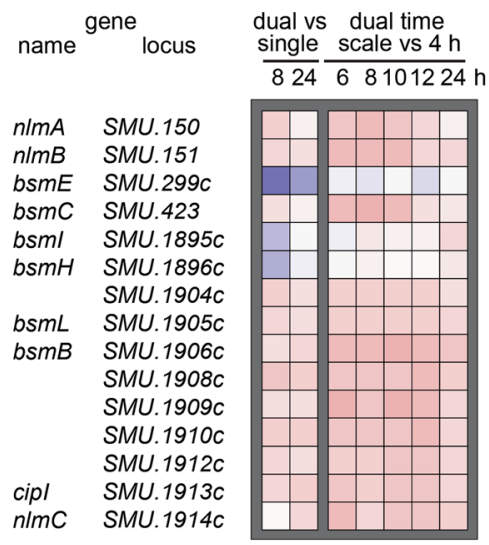

\section{e}

Oxidative stress, chaperones, and Clp proteases

$\begin{array}{ll} & \text { SMU.140 } \\ \text { clpE } & \text { SMU.562 } \\ \text { dpr } & S M U .540 \\ \text { sod } & \text { SMU.629 } \\ & \text { SMU.669c } \\ \text { ahpC } & \text { SMU.764 } \\ \text { ahpF } & \text { SMU.765 } \\ \text { tpx } & \text { SMU.924 } \\ \text { clp } & \text { SMU.956 } \\ \text { nox2 } & \text { SMU.1117 } \\ \text { clpB } & S M U .1425 \\ \text { trxA } & \text { SMU.1869 } \\ \text { groEL } & S M U .1954 \\ \text { groES } & \text { SMU.1955 }\end{array}$

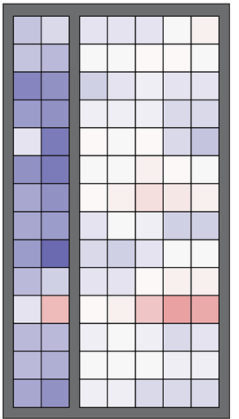

Adhesion and biofilm formation

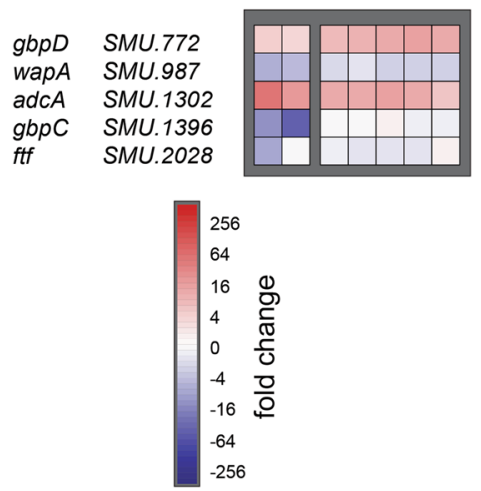

\section{g Carbohydrate metabolism}

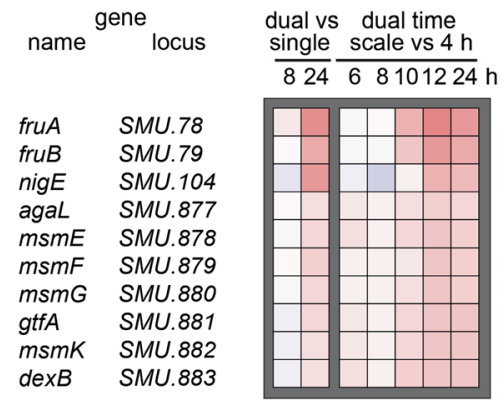

h

\section{PTS systems}

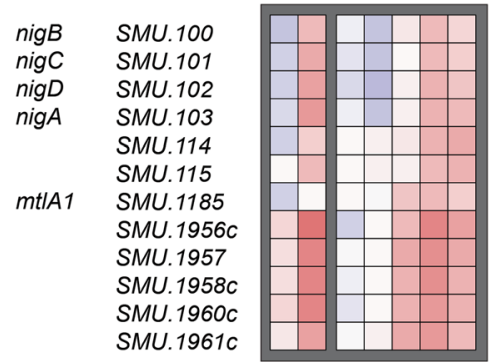

i

\section{Glycogen synthesis}

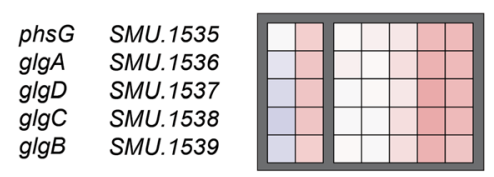

Pyruvate metabolism

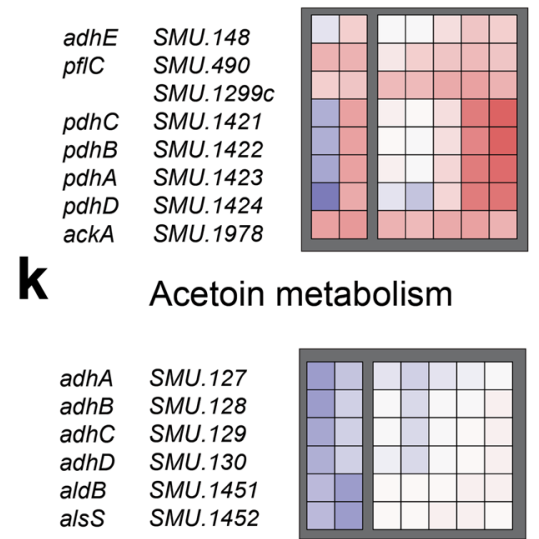

Fig. 4 Transcriptional activity of S. mutans alone and in co-culture with A. act.. Heatmaps show relative gene expression of . mutans after 8 and 24 h of biofilm growth in dual species biofilms with A. act. compared with the expression in single-species biofilms (left two columns) or after $6,8,10,12$ and $24 \mathrm{~h}$ of biofilm growth in dual species biofilms compared with gene expression at $4 \mathrm{~h}$ (right five columns). Selected genes were significantly differentially expressed in at least one condition (FDR $\leq 0.05)$ and are grouped according to function in panel a-k andordered based on their position in the S. mutans chromosome 


\section{Comparison of transcriptomes of A.act. grown alone and} with S. mutans

Comparative analyses of transcriptomes of A. act. grown with $S$. mutans yielded 170 and 97 differentially expressed genes $\left(\log _{2}\right.$ fold change $\leq-2$ and $\geq 2$; $p<0.01$ ) for $8 \mathrm{~h}$ and $24 \mathrm{~h}$, respectively. The main virulence factors of A.act. showed constant high expression irrespective of the tested condition. Leukotoxin was the second most highly expressed $A$. act. transcript (Additional file 5). The tight adherence (tad) locus that is required for the assembly of adhesive pili made up $21 \%$ of the total $A$. act. transcriptome and $f l p-1$ was the most abundant transcript overall. The lsrACFG genes coding for an AI-2 transporter and AI-2 metabolizing enzymes were 8 fold down-regulated in the $8 \mathrm{~h}$ old co-culture (Fig. 5a). The autoinducer synthase LuxS was not differentially expressed. Iron uptake genes were up-regulated in co-culture (Fig. 5b) and changes in anaerobic metabolism likely occured: Fumarate reductase, malate dehydrogenase, formate dehydrogenase (FDH-H) and formate hydrogenlyase complex, and subunits of hydrogenase were induced, while the expression of formate dehydrogenase from the nitrate reductase complex (FDH-N) decreased (Fig. 5c). Genes coding for enzymes involved in nucleotide and DNA metabolism were induced especially in $8 \mathrm{~h}$ old dual species-biofilms (Fig. 5d) and expression of chaperones and heat shock proteins was up-regulated in the 24 h old co-culture. Quinol peroxidase [42] was most strongly (22-fold) induced in both 8 and $24 \mathrm{~h}$ co-culture biofilms (Fig. 5e). This enzyme reduces $\mathrm{H}_{2} \mathrm{O}_{2}$ to water using reducing equivalents from quinol, a component of the respiratory chain. The complement resistance protein ApiA (annotated as putative adhesion/invasion in strain HK1651) and catalase were 6-fold and 11-fold down-regulated at $6 \mathrm{~h}$ in co-culture (Fig. 5e).

\section{Changes in the transcriptomes of S. mutans and A. act. during growth in dual-species biofilms for $24 \mathrm{~h}$}

We found 316 and 309 genes of S. mutans and A. act., respectively, to be differentially expressed during growth in co-culture compared to their expression at $4 \mathrm{~h}$. In $S$. mutans the competence and mutacin related genes were induced from $6 \mathrm{~h}$ onwards (Fig. 4a-d). From $10 \mathrm{~h}$ onwards the reprogramming of metabolism occurred (Fig. 4g-j). In $A$. act. the AI-2 metabolizing enzymes were repressed between 6 and $8 \mathrm{~h}$ (Fig. 5a) and induced at later time-points, in accordance with the up-take of AI-2 in late stationary phase during nutrient depletion. Iron sequestering was most strongly induced between 6 and $8 \mathrm{~h}$ (Fig. 5b). We observed the induction of stress response proteins, chaperones, and the $\mathrm{ClpB}$ protease (Fig. 5e).
Expression of the quorum sensing regulon of $S$. mutans in periodontal pockets

Additional files 8 and 9 show the total and relative number of transcripts from S. mutans and A. act, respectively, in four metatranscriptome studies of the periodontal pocket microbiome [16-18, 43]. Transcripts from both organisms were detected in all studies, although at low abundance in most samples. However, in some samples, 20 to $30 \%$ of all reads mapped to $S$. mutans UA159, showing an extremely strong contribution of this species alone to the community metatranscriptome in some patients. No clear correlation with health or disease was found, yet interestingly, $S$. mutans transcripts had the highest relative abundance in the study of Yost et al. who investigated periodontitis progression [18]. Transcripts from A. act. were rare in all periodontal metatranscriptomes; the maximum value was found in a sample from a healthy individual, where they accounted for $1.4 \%$ of all reads.

A detailed analysis was performed for samples with the highest numbers of reads (between 8347 and 37,798) which were derived from three healthy patients and one with chronic periodontitis [44]. The expression level of the 50 most highly expressed genes of $S$. mutans is shown in Additional file 5. The transcriptional profile depicts an actively growing population: Genes for important metabolic enzymes (pyruvate-formate lyase, glyceraldehyde-3phosphate dehydrogenase, glycerol dehydrogenase, sugar phosphotransferase system, glucosyltransferase, alcoholacetaldehyde dehydrogenase, 6-phosphofructokinase) are expressed, as well as genes encoding RNA polymerase, glucan branching enzyme, surface antigen SpaP and the ATP-dependent protease ClpE. The competence related genes with an average abundance of transcripts $\geq 0.01 \%$ is shown in Table 1 . The most strongly expressed competence related gene was the master regulator of QS in $S$. mutans, the alternative sigma-factor sigX (SMU_1997), which had an average abundance of $0.23 \%$. Additionally, transformasome as well as mutacin related transcripts could be detected. The most strongly expressed bacteriocin was NlmC (mutacin V), which is also the most highly expressed bacteriocin in pure culture. Interestingly, transcripts of comC (SMU_1915), encoding the synthase for the precursor of the mutacin inducing peptide MIP were also detected $(0.06 \%)$. Not all components of the SigX regulon were found, but since the overall transcript level of $S$. mutans was relatively low, this is probably due to lack of sequencing depth. Because of the low absolute number of competence related transcripts in periodontal pockets, it was not possible to calculate the difference in gene expression for transcripts with average abundance $<0.01 \%$. Significant $(\mathrm{FDR}<0.01)$ differential expression was found for $\operatorname{sig} X, \operatorname{com} F A, \operatorname{com} C$ and a hypothetical protein (Additional file 5). The result for $\operatorname{com} C$ should be interpreted with great caution, because in two of the periodontal pocket 


\section{a}

name gene
Al-2 modyfing protein IsrG
aldolase IsrF
ABC tr. IsrC
$A B C$ tr. IsrA'
$A B C$ tr. IsrA

b

Iron transport and storage

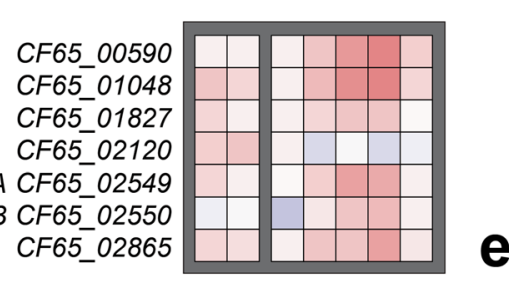

CF65_02865 dual vs dual time

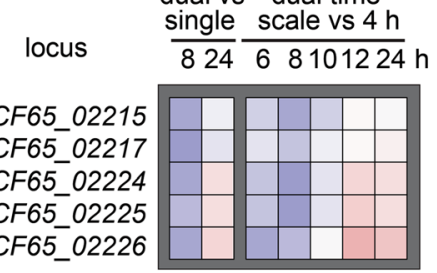

\section{d}

\section{Nucleotide and DNA metabolism}

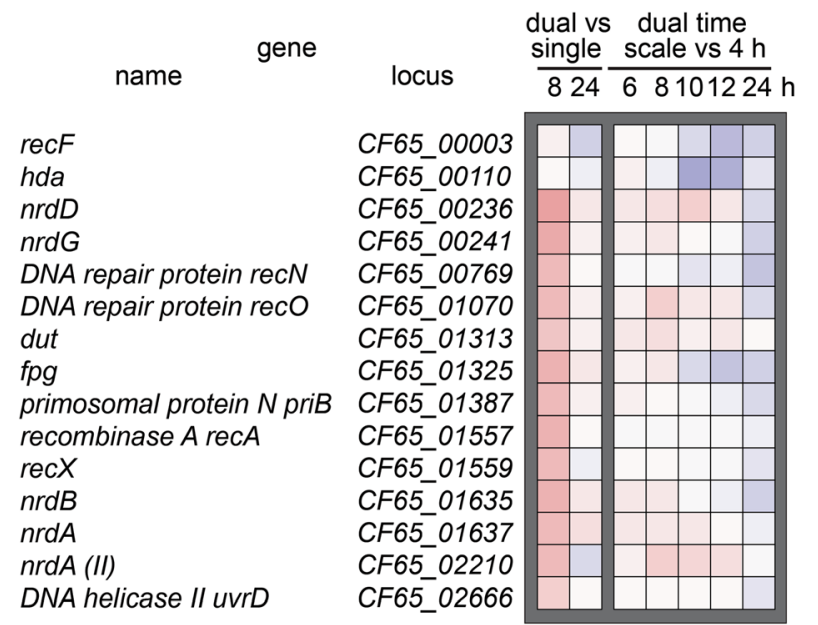

\section{e}

catalase

heat shock protein clpQ chaperone protein dnak heat shock protein dnaJ chaperone protein hscB chaperone protein hscA heat shock protein grpE chaperone protein htpG protease clpB chaperonin groES heat shock protein groEL quinol peroxidase

\section{Stress and chaperones}

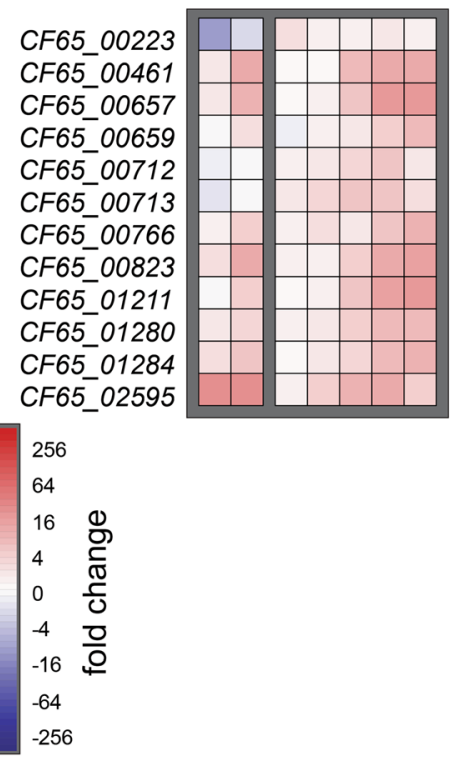

Fig. 5 Transcriptional activity of A. act. alone and in co-culture with S. mutans. Heatmaps show relative gene expression of $A$. act. after 8 and $24 \mathrm{~h}$ of biofilm growth in dual species biofilms with S. mutans compared with the expression in single-species biofilms (left two columns) or after 6 , 8 , 10, 12 and $24 \mathrm{~h}$ of biofilm growth in dual species biofilms compared with gene expression at $4 \mathrm{~h}$ (right five columns). Selected genes are grouped according to function in panel a-e and ordered based on their position in the $A$. act. chromosome

samples (AU_8 and AI_19) no transcript of $\operatorname{com} C$ was found, while in the other two (AU_9 and AU_12) their frequency was low $(0.212 \%$ and $0.010 \%$ of all transcripts). However, it is now known that competence is induced by SigX, while CSP, the product of processed ComC, controls mutacin synthesis only and was therefore renamed MIP (mutacin inducing peptide) [20]. From all competence related genes, sig $X$ was most strongly differentially enriched ( $\log 2$ fold change 4.159, FDR $<0.001)$. A comparison of the expression of the QS related genes of $S$. mutans in dual and single species biofilms and in periodontal pockets based on percent of the respective transcript in comparison to the total amount of transccripts from this sample (Fig. 6) shows that biological replicates of in vitro cultures were almost identical, while periodontal communities showed large differences in the expression of QS related genes. However, sig $X$ was not expressed in pure culture of $S$. mutans, neither during exponential growth nor in stationary phase, but was expressed in all four periodontal pockets, sometimes at levels similar 
Table 1 The expression of the SigX regulon of $S$. mutans in the periodontal community (sample AU_08, AU_09, AU_12, AU_19 from (39)

\begin{tabular}{|c|c|c|}
\hline Locus tag & Mean percentage & Product \\
\hline SMU_1997 & 0.23524 & $\begin{array}{l}\text { SigX, competence inducing alternative } \\
\text { sigma-factor }\end{array}$ \\
\hline SMU_1914C & 0.12851 & NImC, bacteriocin \\
\hline SMU_1978 & 0.12642 & ComYA, transformasome component \\
\hline SMU_837 & 0.05622 & putative reductase, competence related \\
\hline SMU_1915 & 0.05583 & $\begin{array}{l}\text { ComC, competence stimulating } \\
\text { peptide precursor }\end{array}$ \\
\hline SMU_2085 & 0.05410 & RecA, recombination protein \\
\hline SMU_1904C & 0.05354 & bacteriocin related \\
\hline SMU_498 & 0.05178 & ComFA, late competence protein \\
\hline SMU_2086 & 0.05098 & $\begin{array}{l}\text { CinA, competence and damage } \\
\text { inducible protein }\end{array}$ \\
\hline SMU_1912C & 0.03916 & bacterocin related \\
\hline SMU_1001 & 0.03679 & $\begin{array}{l}\text { Smf, DNA processing protein, } \\
\text { competence related }\end{array}$ \\
\hline SMU_1910C & 0.03512 & hypothetical protein \\
\hline SMU_1987 & 0.03450 & $\begin{array}{l}\text { ATP-binding protein ComYA; late } \\
\text { competence gene }\end{array}$ \\
\hline SMU_539C & 0.03360 & signal peptidase type IV \\
\hline SMU_61 & 0.03270 & putative transcriptional regulator \\
\hline SMU_836 & 0.03171 & hypothetical protein \\
\hline SMU_1913C & 0.03148 & immunity protein, BLpL-like \\
\hline SMU_423 & 0.02861 & hypothetical protein \\
\hline SMU_151 & 0.02800 & hypothetical protein \\
\hline SMU_1895C & 0.02739 & hypothetical protein \\
\hline SMU_1896c & 0.02192 & hypothetical protein \\
\hline SMU_1917 & 0.01765 & $\begin{array}{l}\text { response regulator of the competence } \\
\text { regulon, ComE; }\end{array}$ \\
\hline SMU_626 & 0.01635 & competence protein \\
\hline SMU_644 & 0.01267 & $\begin{array}{l}\text { competence protein/transcription } \\
\text { factor }\end{array}$ \\
\hline
\end{tabular}

Only genes with an average abundance $>0.01 \%$ are shown

(sample pp_8) or even higher (sample pp_09) than those in dual-species biofilms. Since SigX is the master regulator of both competence and bacteriocin synthesis, we therefore hypothesize that $S$. mutans is genetically competent in periodontal pockets.

\section{Discussion}

We found that cell-cell communication in S. mutans was induced by the phylogenetically distantly related species $A$. act.. Induction occurred by a component in the culture medium that was only produced in live dual-species biofilms and that was not produced any more upon deletion of comS of S. mutans. Thus, production of the ComS prepeptide was induced in coculture, similarly as in dual-species biofilms between
S. mutans and the fungus C. albicans [21]. Leukotoxin and fimbriae are not likely to be involved since both the highly virulent strain HK1651 of $A$. act. and the "smooth" type strain induced QS in S. mutans. On the other hand, complex media containing components known to block the Opp permease required for import of the extracellular XIP [39] completely inhibited activation of SigX. This observation shows that the complete autocatalytic cycle of ComS synthesis, processing, export and import was activated in dual species biofilms. Since the stimulating factor is only produced in co-culture, it must be the result of an interaction between the two species in the biofilm. It is unclear if physical contact is required for the primary activation, yet since sterile filtered culture supernatants can activate SigX, this seems unlikely.

Deletion of the histidine kinase LiaS of $S$. mutans (Smu.486) resulted in a strain that could not be induced by $A$. act. or by synthetic XIP to express $\operatorname{sigX}$. LiaS is a sensor histidine kinase which represses glucan binding protein $\mathrm{C}(g b p C)$ transcription and induces synthesis of mutacin IV (cipB) [45]. In our transcriptome data, $g b p C$ was down-regulated, and mutacin IV was upregulated in dual-species biofilms with $A$. act.. Recently, the LiaR regulon was shown to be comprised of three genes, namely Smu.753, Smu.1727 and Smu.2084c [46]. None of them was differentially expressed in dual species biofilms, which makes it very unlikely that sensing of a specific stimulus was responsible for the effect of LiaS on $\operatorname{sig} X$ induction. Since our data show that import of externally added, synthetic XIP was not possible in a $\Delta l i a S$ mutant, we hypothesize that the cell membrane of this mutant was not permeable for XIP any more. Therefore we further hypothesize that exported XIP cannot re-enter the cell and the positive feed-back loop for XIP synthesis cannot be triggered.

We show that mucin inhibited QS induction in dualspecies biofilms in a concentration dependent way. Mucins are glycoproteins contributing to the antimicrobial activity of saliva [47], to which we can now add their ability to inhibit the QS of $S$. mutans. This might be caused by blockage of the Opp permease [39] that can then not import XIP anymore. Mucin concentration can be decreased in oral biofilms by streptococci [48] to levels which no longer block signaling.

S. mutans down-regulated the expression of genes encoding chaperones and genes involved in oxidative stress in coculture with $A$. act. suggesting that $A$. act. protected $S$. mutans from oxidative stress. Oxygen may have been depleted by the aerobic respiration of $A$. act.. Surprisingly, its catalase gene was down-regulated in co-culture, and stress and chaperone related genes were up-regulated, in contrast to $S$. mutans. The strong up-regulation of the quinol peroxidase gene also indicates oxidative stress for 


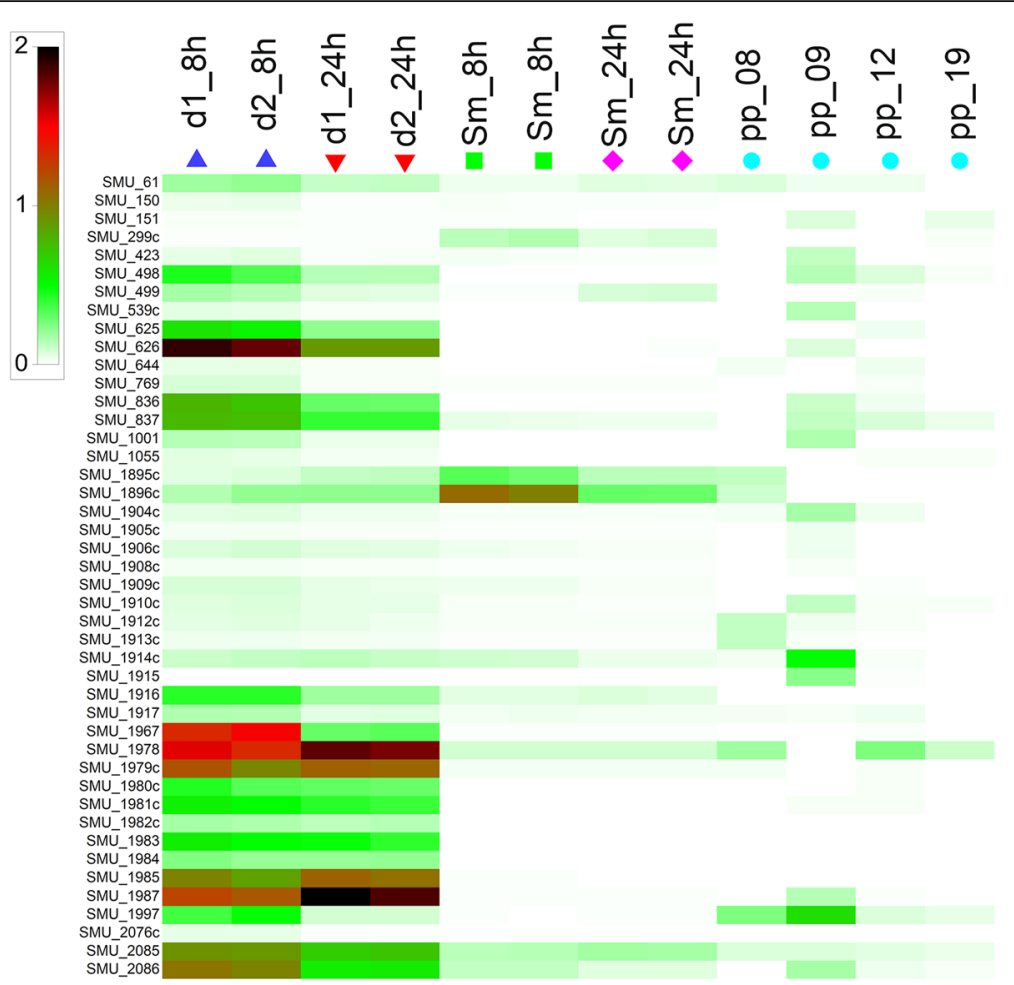

Fig. 6 Expression of the QS regulon of S. mutans in dual culture, single culture, and in periodontal pockets. Relative abundance of transcripts of $S$. mutans (\% of total transcripts) after 8 (d1_8h, d2_8h) and $24 \mathrm{~h}\left(\mathrm{~d} 1 \_24 \mathrm{~h}, \mathrm{~d} 2 \_24 \mathrm{~h}\right)$ in co-culture with $\mathrm{A}$. act., in single culture after $8 \mathrm{~h}$ (Sm_8h) and $24 \mathrm{~h}$ (Sm_24h), and in periodontal pockets (pp_08, pp_9, pp_12, pp_19). Sample pp_08 was derived from an individual with chronic periodontitis, while the other three periodontal pocket samples were derived from healthy individuals. RNAseq data from laboratory cultures are shown for two biological replicates. Metatranscriptome data from [16]

A.act.. These apparent inconsistencies might be caused by oxygen gradients in biofilms [49] and by differences in the localization of the cells within the biofilm [24]. In dual species biofilms we observed tight balls of $A$. act. embedded in a thick layer of $S$. mutans cells (Additional file 10). Inside the balls, conditions were most likely anaerobic. Moreover, competition for iron uptake may have stressed the cells especially within the balls. Consistent with these observations, fermentation pathway enzymes were up-regulated in A.act. in co-culture.

A.act. strongly expressed virulence genes both in the presence and absence of $S$. mutans. It was noteworthy that on average $20 \%$ of transcriptional activity of this organism was devoted to the tad locus producing adhesive fimbriae. The gene encoding leukotoxin was the second most highly expressed gene overall. Thus, in our experimental setup A. act. most probably maintained an extremely virulent form. Interestingly, the two genes involved in immune response evasion, encoding catalase KatA and the complement resistance protein ApiA, were repressed in our coculture, while they were induced in co-culture with $S$. gordonii [28]. This suggests that A.act. is more susceptible to the host immune defense in the presence of $S$. mutans, which might explain clinical studies which have found a negative relationship between caries and aggressive periodontitis [50].

Our data show that $S$. mutans probably produces mutacins and is genetically competent in vivo, indicated by the relatively large number of transcripts of $\operatorname{sig} X$ as well as of many components of the SigX regulon. Moreover, the expression of $\operatorname{com} C$, the gene encoding the synthase for the mutacin inducing peptide, was detected in dual species biofilms and in periodontal pockets, but not in biofilms of $S$. mutans alone, neither during exponential growth nor in the stationary phase. Apparently the QS system of $S$. mutans can be induced not only by $C$. albicans and $A$. act., but also by interaction with diverse members of the oral microbiome. The exact mechanism through which this occurs remains to be elucidated. The data suggest that QS of $S$. mutans is not so much a mechanism for orchestrating gene expression of its own population according to cell density, but a mechanism for adjusting its phenotype to the presence and activity of the oral microbiome. Killing competitors and using their DNA for genetic adaptation provides a powerful survival mechanism in complex communities, but is meaningless in pure culture. 


\section{Conclusion}

The complete quorum sensing regulon of $S$. mutans was induced by $A$. act. by an unknown mechanism which required the presence of ComS, the synthase for the XIP prepeptide. A. act. grew in a highly virulent form but down-regulated the genes important for escape from the host immune response in co-culture with $S$. mutans. Transformasome and mutacin genes of $S$. mutans were expressed in periodontal pockets, suggesting that its quorum sensing regulon is active in vivo. Because polymicrobial communities harboring streptococci and Aggregatibacter spp. are causing oral infectious diseases, the observed interactions may have an important role in the dysbiosis of such communities.

\section{Additional files}

Additional file 1: Strains and primers used in the study. (DOCX $26 \mathrm{~kb}$ )

Additional file 2: Biofilm formation of S. mutans in single and dual-species biofilms with $A$. act. on different media. (a) CDMS, chemically defined medium with sucrose, (b) THBYS, Todd-Hewitt broth with yeast extract and sucrose, (c) YNBB2, biofilm medium (d) AS, artificial saliva, (e) modified AS, (mucin was omitted and haemin was replaced with $1.2 \mu \mathrm{M} \mathrm{FeCl}$ ). All media were additionally buffered and supplemented with sucrose. Biofilm mass was determined by crystal violet staining. See methods for details. (TIF $281 \mathrm{~kb}$ )

Additional file 3: Activation of the sigX promoter of S. mutans in single and dual-species biofilms with A. act on different media. (a) CDMS, chemically defined medium with sucrose, (b) THBYS, Todd-Hewitt broth with yeast extract and sucrose, (c) YNBB2, biofilm medium (d) AS, artificial saliva, (e) modified AS, (mucin was omitted and haemin was replaced with $1.2 \mu \mathrm{M} \mathrm{FeCl}$ ). All media were additionally buffered and supplemented with sucrose. Activation was determined as fluorescence intensity of $\mathrm{SMP}_{\text {sig }} \mathrm{GFP}$, a gfp-reporter for sigX expression in S. mutans. See methods for details. (TIF $273 \mathrm{~kb}$ )

Additional file 4: Influence of mucin on sigX fluorescence (A), biofilm formation and sigX activation by A.act. HK1651 (B,C), and analysis of sequencing depths (D-F). (A) Fluorescence of $S$. mutans reporter strain SMP $_{\text {sig }}$ GFP co-cultured with $A$. act. in dual-species biofilms on YNBB2 medium supplemented with the indicated amount of mucin. (B) Biofilm biomass and (C) fluorescence of S. mutans SMUsigXGFP activated by by culture supernatants from single- and dual-species biofilms with A.act. HK1651. (D-E) Sequencing depth. Mean $\log _{2}$ transformed counts per gene per million reads (CPM) for all detected S. mutans (D) and A. act. (E) genes. As a cut-off for expression, 10 CPM was chosen. (F) Percentage and number of genes above and below cut-off and with CPM $=0$. (TIF $325 \mathrm{~kb}$ )

Additional file 5: Sheet 1: Overview of biofilm sequencing results. Sheet 2: Small RNAs expressed by S. mutans in dual-species biofilms with A.act. Sheet 2: Top 20 genes of $S$. mutans expressed in dual-species biofilms with $A$. act.. Sheet 3: Top 20 genes of $A$. act. expressed in dual-species biofilms with S. mutans. Sheet 4: Top 50 most abundant transcripts of $S$. mutans in the periodontal community (sample AU_08, AU_09, AU_12, $A U_{-}$19). Sheet 5: Relative abundace of transcripts of the SigX regulon of $S$. mutans in the periodontal community (sample AU_8, AU_9, AU_12, AU_19). Sheet 6: Differentially expressed genes of the SigX regulon of S. mutans (periodontal pocket versus pure culture at $8 \mathrm{~h}$ ). Sheet 7: Relative abundance (\%) of transcripts of S. mutans in four periodontal pocket samples (sample AU_08, AU_09, AU_12, AU_19) Sheet 8: Relative abundance (\%) of A. act. genes expressed in dual-species biofilms. (XLSX $530 \mathrm{~kb}$ )

Additional file 6: C-means clustering of differentially expressed genes of S. mutans from dual-species biofilms with A. act. during growth in comparison to $4 \mathrm{~h}$. (a) Calculation of minimum centroid distance for a range of cluster numbers. On this basis, 8 clusters were defined (marked with a dashed line). (b) PCA analysis of clusters. Two clear groups are formed. Blue lines indicate relationship between clusters. Changes in expression of genes in group 1 (c), group 2 (d) and cluster 1 to 8 (e) are shown. (TIF $934 \mathrm{~kb}$ )

Additional file 7: C-means clustering of differentially expressed genes of $A$. act. from dual-species biofilms with $S$. mutans during growth in comparison to $4 \mathrm{~h}$. (a) Calculation of minimum centroid distance for a range of cluster numbers. On this basis, 8 clusters were defined (marked with a dashed line). (b) PCA analysis of clusters. Two clear groups are formed. Blue lines indicate relationship between clusters. Changes in expression of genes in group 1 (c), group 2 (d) and cluster 1 to 8 (e) are shown. (TIF $807 \mathrm{~kb}$ )

Additional file 8: Relative abundance of transcripts from S. mutans in metatranscriptomes from periodontal pocket samples. Data are derived from $[16-18,43]$. Reads are shown as absolute number of reads in the respective sample (left) and in \% of total reads (right). (JPG $1822 \mathrm{~kb}$ )

Additional file 9: Relative abundance of transcripts from A. act. in metatranscriptomes from periodontal pocket samples. Data are derived from $[16-18,43]$. Reads are shown as absolute number of reads in the respective sample (left) and in \% of total reads (right). (JPG $1820 \mathrm{~kb}$ )

Additional file 10: Microscopic analysis of dual-species biofilms. Dualspecies biofilms of $A$. act. and S. mutans reporter strain SMP sigx GFP. (a) S. mutans (green) expressed Gfp, A. act. (blue) was counterstained with 4,6-diamidino-2-phenylindole (DAPI). A. act. formed "ball-like" clusters of tightly packed cells. (b) Ball-like clusters at higher magnification. (c) Gram staining of biofilms. S. mutans (left) appears violet and A. act. (right) is stained pink. (d) "Ball-like" clusters of cells at high magnification. Scale bars (a) $20 \mu \mathrm{m}$; (b) $100 \mu \mathrm{m}$; (c) $50 \mu \mathrm{m}$; (e) $6 \mu \mathrm{m}$. (a) and (b) were obtained by fluorescence microscopy, (c) by light microscopy, (e) by scanning electron microscopy. (TIF $3732 \mathrm{~kb}$ )

\section{Abbreviations}

A. act: Aggregatibacter actinomycetemcomitans; AS: Artificial saliva; CDMS: Chemically defined medium with sucrose; ComR: Response regulator induced by XIP; ComS: Autoinducer synthase for the XIP prepeptide; CSP: Competence stimulating peptide; EPS: Extracellular polysaccharide; GFP: Green fluorescent protein; HK: Histidine kinase; MIP (former (SP): Mutacin inducing peptide; PCA: Principal component analysis; qPCR: Quantitative PCR; q-RT-PCR: Quantitative reverse transcription PCR; QS: quorum sensing; S.: Streptococcus; SigX: alternative sigma-factor; THBY: Todd-Hewitt broth with yeast extract; THBYS: Todd-Hewitt broth with yeast extract and sucrose; XIP: SigX inducing peptide; XIP: sigX-inducing peptide; YNBB2: biofilm medium

\section{Acknowledgments}

We are grateful to Georg Conrads, Catherine M. Levesque, and Michael Reck for providing bacterial strains.

\section{Funding}

JT was financed by the German Research Organisation (DFG) through the Transregio-SFB TRR-51 Roseobacter. ZD gratefully acknowledges support through the COMBACTE grant. The funders had not role in study design, data collection and interpretation or the decision to submit the work for publication.

\section{Availability of data and materials}

Sequencing data are available at Gene Expression Omnibus under accession number GSE75019. All data supporting our findings are contained in the manuscript and the eight additional files submitted as supplementary material.

\section{Authors'contributions}

SPS conducted the experiments, analysed the data, and wrote the manuscript draft. ZD and JT performed bioinformatical and statistical analyses. SPS, ZD and JT prepared the figures. MJ and SB prepared sequencing libraries and processed the raw sequences. MR provided scanning electron micrographs. IWD and HS designed the study and supervised SPS. IWD finalized the manuscript. All authors read and approved the final manuscript.

\section{Competing interests}

The authors declare that they have no competing interests. 


\section{Consent for publication}

Not applicable.

\section{Ethics approval and consent to participate} Not applicable.

\section{Publisher's Note}

Springer Nature remains neutral with regard to jurisdictional claims in published maps and institutional affiliations.

\section{Author details}

${ }^{1}$ Microbial Communication, Helmholtz-Center for Infection Research, Braunschweig, Germany. ${ }^{2}$ Genome Analytics, Helmholtz Centre for Infection Research, Braunschweig, Germany. ${ }^{3}$ Central Facility for Microscopy, Helmholtz Centre for Infection Research, Braunschweig, Germany. ${ }^{4}$ Present address: Hannover Medical School (MHH), Hannover, Germany.

Received: 17 November 2016 Accepted: 10 March 2017

Published online: 20 March 2017

\section{References}

1. Clarke JK. On the bacterial factor in the etiology of dental caries. British journal of experimental pathology. 1924;5:141-7.

2. Loesche WJ. Role of Streptococcus mutans in human dental decay. Microbiol Rev. 1986;50:353-80.

3. Simon-Soro A, Belda-Ferre $P$, Cabrera-Rubio R, Alcaraz LD, Mira A A tissue-dependent hypothesis of dental caries. Caries Res. 2013;47:591-600. doi:10.1159/000351663.

4. Simon-Soro A, Guillen-Navarro M, Mira A. Metatranscriptomics reveals overall active bacterial composition in caries lesions. J Oral Microbiol. 2014;6:25443.

5. Teng F, Yang F, Huang S, Bo C, Xu ZZ, Amir A, Knight R, Ling J, Xu J. Prediction of early childhood caries via spatial-temporal variations of oral microbiota. Cell Host Microbe. 2015;18:296-306. doi:10.1016/j.chom.2015.08.005.

6. Simon-Soro A, Mira A. Solving the etiology of dental caries. Trends Microbiol. 2015;23:76-82. doi:10.1016/j.tim.2014.10.010.

7. Guo L, McLean JS, Yang Y, Eckert R, Kaplan CW, Kyme P, Sheikh O, Varnum B, Lux R, Shi W, He X. Precision-guided antimicrobial peptide as a targeted modulator of human microbial ecology. Proc Natl Acad Sci U S A. 2015;112:7569-74.

8. Thompson JA, Oliveira RA, Xavier KB. Chemical conversations in the gut microbiota. Gut Microbes. 2016;7:163-70. doi:10.1080/19490976.2016.1145374.

9. Guo L, He X, Shi W. Intercellular communications in multispecies oral microbial communities. Front Microbiol. 2014;5:328. doi:10.3389/fmicb.2014.00328.

10. Cook LC, Federle MJ. Peptide pheromone signaling in Streptococcus and Enterococcus. FEMS Microbiol Rev. 2014;38:473-92. doi:10.1111/1574-6976.12046.

11. Cook LC, LaSarre B, Federle MJ. Interspecies communication among commensal and pathogenic streptococci. MBio. 2013;4(4):e00382-13. doi:10.1128/mBio.00382-13.

12. Aggarwal C, Jimenez JC, Lee H, Chlipala GE, Ratia K, Federle MJ. Identification of quorum-sensing inhibitors disrupting signaling between Rgg and short hydrophobic peptides in streptococci. MBio. 2015;6:e00393-15.

13. Parashar V, Aggarwal C, Federle MJ, Neiditch MB. Rgg protein structurefunction and inhibition by cyclic peptide compounds. Proc Natl Acad Sci U S A. 2015;112:5177-82. doi:10.1073/pnas.1500357112.

14. Chang JC, Jimenez JC, Federle MJ. Induction of a quorum sensing pathway by environmental signals enhances group A streptococcal resistance to lysozyme. Mol Microbiol. 2015;97:1097-113. doi:10.1111/mmi.13088.

15. Jorth P, Trivedi U, Rumbaugh K, Whiteley M. Probing bacterial metabolism during infection using high-resolution transcriptomics. J Bacteriol. 2013; 195:4991-8. doi:10.1128/JB.00875-13.

16. Szafranski SP, Deng Z-L, Tomasch J, Jarek M, Bhuju S, Meisinger C, Kühnisch J, Sztajer H, Wagner-Döbler I. Functional biomarkers for chronic periodontitis and insights into the roles of Prevotella nigrescens and Fusobacterium nucleatum: a metatranscriptome analysis. Nature Biofilms and Microbiomes. 2015;1. doi:10. 1038/npjbiofilms.2015.17.

17. Duran-Pinedo AE, Chen T, Teles R, Starr JR, Wang X, Krishnan K, Frias-Lopez $J$. Community-wide transcriptome of the oral microbiome in subjects with and without periodontitis. ISME J. 2014;8:1659-72. doi:10.1038/ismej.2014.23.

18. Yost S, Duran-Pinedo AE, Teles R, Krishnan K, Frias-Lopez J. Functional signatures of oral dysbiosis during periodontitis progression revealed by microbial metatranscriptome analysis. Genome Med. 2015;7:27. doi:10.1186/s13073-015-0153-3.
19. Mashburn-Warren L, Morrison DA, Federle MJ. A novel double-tryptophan peptide pheromone controls competence in Streptococcus spp. via an Rgg regulator. Mol Microbiol. 2010;78:589-606. doi:10.1111/j.1365-2958.2010.07361.x.

20. Reck M, Tomasch J, Wagner-Dobler I. The alternative sigma factor SigX controls bacteriocin synthesis and competence, the Two quorum sensing regulated traits in streptococcus mutans. PLoS Genet. 2015;11:e1005353. doi:10.1371/journal.pgen.1005353.

21. Sztajer H, Szafranski SP, Tomasch J, Reck M, Nimtz M, Rohde M, WagnerDobler I. Cross-feeding and interkingdom communication in dual-species biofilms of Streptococcus mutans and Candida albicans. ISME J. 2014;8: 2256-71. doi:10.1038/ismej.2014.73.

22. Kononen E, Muller HP. Microbiology of aggressive periodontitis. Periodontol. 2014;65:46-78. doi:10.1111/prd.12016.

23. Hajishengallis G, Lamont RJ. Beyond the red complex and into more complexity: the polymicrobial synergy and dysbiosis (PSD) model of periodontal disease etiology. Mol Oral Microbiol. 2012;27:409-19. doi:10.1111/j.2041-1014.2012.00663.x.

24. Mark Welch JL, Rossetti BJ, Rieken CW, Dewhirst FE, Borisy GG. Biogeography of a human oral microbiome at the micron scale. Proc Natl Acad Sci U S A. 2016;113:E791-800. doi:10.1073/pnas.1522149113.

25. Kolenbrander PE. Multispecies communities: interspecies interactions influence growth on saliva as sole nutritional source. Int J Oral Sci. 2011;3:49-54. doi:10.4248/IJOS11025.

26. Stacy A, McNally L, Darch SE, Brown SP, Whiteley M. The biogeography of polymicrobial infection. Nat Rev Microbiol. 2016;14:93-105. doi:10.1038/nrmicro.2015.8.

27. Stacy A, Everett J, Jorth P, Trivedi U, Rumbaugh KP, Whiteley M. Bacterial fight-and-flight responses enhance virulence in a polymicrobial infection. Proc Natl Acad Sci U S A. 2014;111:7819-24. doi:10.1073/pnas.1400586111.

28. Ramsey MM, Whiteley M. Polymicrobial interactions stimulate resistance to host innate immunity through metabolite perception. Proc Natl Acad Sci U S A. 2009;106:1578-83. doi:10.1073/pnas.0809533106.

29. Lemme A, Grobe L, Reck M, Tomasch J, Wagner-Dobler I. Subpopulation-specific transcriptome analysis of competencestimulating-peptide-induced Streptococcus mutans. J Bacteriol. 2011;193: 1863-77. doi:10.1128/JB.01363-10.

30. Wong $L$, Sissons $C$. A comparison of human dental plaque microcosm biofilms grown in an undefined medium and a chemically defined artificial saliva. Arch Oral Biol. 2001;46:477-86.

31. van de Rijn I, Kessler RE. Growth characteristics of group a streptococci in a new chemically defined medium. Infect Immun. 1980;27:444-8.

32. Lau PC, Sung CK, Lee JH, Morrison DA, Cvitkovitch DG. PCR ligation mutagenesis in transformable streptococci: application and efficiency. J Microbiol Methods. 2002;49:193-205.

33. Periasamy S, Kolenbrander PE. Aggregatibacter actinomycetemcomitans builds mutualistic biofilm communities with Fusobacterium nucleatum and Veillonella species in saliva. Infect Immun. 2009;77:3542-51. doi:10.1128/IAl.00345-09.

34. Langmead B, Salzberg SL. Fast gapped-read alignment with Bowtie 2. Nat Methods. 2012;9:357-9. doi:10.1038/nmeth.1923.

35. Robinson MD, McCarthy DJ, Smyth GK. edgeR: a Bioconductor package for differential expression analysis of digital gene expression data. Bioinformatics. 2010;26:139-40. doi:10.1093/bioinformatics/btp616.

36. Kumar L, Futschik E. Mfuzz: a software package for soft clustering of microarray data. Bioinformation. 2007:2:5-7.

37. McClure R, Balasubramanian D, Sun Y, Bobrovskyy M, Sumby P, Genco CA, Vanderpool CK, Tjaden B. Computational analysis of bacterial RNA-Seq data. Nucleic Acids Res. 2013;41:e140. doi:10.1093/nar/gkt444.

38. Kolde R. pheatmap: Pretty Heatmaps. R package version 0.7.7., version. 2013.

39. Son M, Ahn SJ, Guo Q, Burne RA, Hagen SJ. Microfluidic study of competence regulation in Streptococcus mutans: environmental inputs modulate bimodal and unimodal expression of comX. Mol Microbiol. 2012;86:258-72. doi:10.1111/j.1365-2958.2012.08187.x.

40. Levesque CM, Mair RW, Perry JA, Lau PC, Li YH, Cvitkovitch DG. Systemic inactivation and phenotypic characterization of two-component systems in expression of Streptococcus mutans virulence properties. Lett Appl Microbiol. 2007:45:398-404. doi:10.1111/j.1472-765X.2007.02203.X.

41. Haubek D, Johansson A. Pathogenicity of the highly leukotoxic JP2 clone of Aggregatibacter actinomycetemcomitans and its geographic dissemination and role in aggressive periodontitis. J Oral Microbiol. 2014;6. doi:10.3402/jom.v6.23980. 
42. Takashima E, Konishi K. Characterization of a quinol peroxidase mutant in Aggregatibacter actinomycetemcomitans. FEMS Microbiol Lett. 2008;286:66-70. doi:10.1111/j.1574-6968.2008.01253.x.

43. Jorth P, Turner KH, Gumus P, Nizam N, Buduneli N, Whiteley M. Metatranscriptomics of the human oral microbiome during health and disease. MBio. 2014:5:e01012-4. doi:10.1128/mBio.01012-14.

44. Szafranski SP, Wos-Oxley ML, Vilchez-Vargas R, Jauregui R, Plumeier I, Klawonn F, Tomasch J, Meisinger C, Kühnisch J, Sztajer H, Pieper DH, Wagner-Döbler I. High-resolution taxonomic profiling of the subgingival microbiome for biomarker discovery and periodontitis diagnosis. Appl Environ Microbiol. 2015;81:1047-58. doi:10.1128/AEM.03534-14.

45. Chong P, Drake L, Biswas I. LiaS regulates virulence factor expression in Streptococcus mutans. Infect Immun. 2008;76:3093-9. doi:10.1128//AI.01627-07.

46. Shankar M, Mohapatra SS, Biswas S, Biswas I. Gene regulation by the LiaSR Two-component system in streptococcus mutans. PLoS One. 2015;10: e0128083. doi:10.1371/journal.pone.0128083.

47. HW v't, Veerman EC, Nieuw Amerongen AV, Ligtenberg AJ. Antimicrobial defense systems in saliva. Monogr Oral Sci. 2014;24:40-51. doi:10.1159/000358783.

48. van der Hoeven JS, van den Kieboom CW, Camp PJ. Utilization of mucin by oral Streptococcus species. Antonie Van Leeuwenhoek. 1990;57:165-72.

49. Wessel AK, Arshad TA, Fitzpatrick M, Connell JL, Bonnecaze RT, Shear JB, Whiteley M. Oxygen limitation within a bacterial aggregate. MBio. 2014;5:e00992. doi:10.1128/mBio.00992-14.

50. Sulugodu RS. Low levels of caries in aggressive periodontitis: a literature review. Saudi Dent J. 2014;26:47-9.

\section{Submit your next manuscript to BioMed Central and we will help you at every step:}

- We accept pre-submission inquiries

- Our selector tool helps you to find the most relevant journal

- We provide round the clock customer support

- Convenient online submission

- Thorough peer review

- Inclusion in PubMed and all major indexing services

- Maximum visibility for your research

Submit your manuscript at www.biomedcentral.com/submit 